\title{
Elongational Viscosity of LDPEs and Polystyrenes Using Entrance Loss Data
}

\author{
K. Walczak, ${ }^{1}$ M. Gupta, ${ }^{1}$ K.A. Koppi, ${ }^{2}$ J. Dooley, ${ }^{2}$ M.A. Spalding ${ }^{2}$ \\ ${ }^{1}$ Mechanical Engineering-Engineering Mechanics Department, Michigan Technological University, \\ Houghton, Michigan 49931
}

${ }^{2}$ The Dow Chemical Company, Midland, Michigan 48667

\begin{abstract}
For two low-density polyethylenes and two polystyrenes, axisymmetric and planar elongational viscosities are estimated using entrance loss data from capillary and slit rheometers, respectively. The elongational viscosity is estimated by optimizing the values of various parameters in the Sarkar-Gupta elongational viscosity model such that the entrance loss predicted by a finite element simulation agrees with the corresponding experimental data. The predicted entrance loss is in good agreement with the experimental data at high flow rates. The difference in the experimental and predicted entrance loss at lower flow rates might have been caused by large error in the experimental data in this range. POLYM. ENG. SCI., 48:223-232, 2008. () 2007 Society of Plastics Engineers
\end{abstract}

\section{INTRODUCTION}

Owing to their long chain molecules, polymers exhibit very complex rheological behavior. Some of the phenomena due to this macromolecular structure of polymers such as die swell and rod climbing in shear flow [1] are unique to polymers and are not experienced by any nonpolymeric fluid. One of these peculiar characteristics of polymers is their high resistance to elongational deformation. Even though the highly entangled long chain molecules of polymers can slip past each other rather easily in a shear flow leading to their shear-thinning behavior, the long chain molecules do not align and flow as easily in elongational flows. Therefore, elongational viscosity of polymers, which characterizes the resistance of a polymer to elongational deformation, is very high.

Trouton [2] was the first person to measure the elongational viscosity of a fluid. Using various methods such as torsion of a bar and sagging of a beam, Trouton estimated

Correspondence to: M. Gupta; e-mail: mahesh@mtu.edu

Contract grant sponsor: National Science Foundation; contract grant number: DMI-0200091.

DOI 10.1002/pen.20818

Published online in Wiley InterScience (www.interscience.wiley.com). (C) 2007 Society of Plastics Engineers that for axisymmetric flows the elongational viscosity of materials, such as pitch and wax, is three times their shear viscosity. The ratio of axisymmetric elongational viscosity to shear viscosity, which is called Trouton ratio, is 3 for most Newtonian fluids. It can also be shown analytically that the axisymmetric elongational viscosity for Newtonian fluids should be three times the shear viscosity and that the elongational viscosity for planar flows should be four times the shear viscosity.

For polymeric fluids, the Trouton ratio is 3 and 4 for axisymmetric and planar flows, respectively, only at low elongation rates within what is known as the Newtonian region of polymeric viscosity. Direct measurement of elongational viscosity by various techniques such as uniaxial extension, lubricated compression, fiber spinning, bubble collapse, stagnation flow, etc. [1, 3] shows that the ratio of elongational viscosity to shear viscosity for polymers at intermediate elongation rates is typically much higher than its value for Newtonian fluids. Unfortunately, at higher elongation rates it is extremely difficult to maintain a purely elongational steady flow. Therefore, direct measurement techniques can only be used to determine elongational viscosity at relatively small elongation rates (less than $10 \mathrm{~s}^{-1}$ ). This limitation on elongation rate is particularly restrictive because in most polymer processing techniques, such as extrusion and injection molding, elongation rate is typically in the range of 1000 $100,000 \mathrm{~s}^{-1}$. Therefore, the experimental data from various techniques for direct measurement of elongational viscosity is not adequate for simulation of most polymer processing techniques.

Due to the limitation of direct measurement techniques, the entrance flow method has been used in the past with various degrees of sophistication to estimate the elongational viscosity of polymers. A highly elongationdominated flow can be conveniently obtained near an abrupt contraction in a channel. This highly elongationdominated flow is referred to as entrance flow. Because of the high elongational viscosity of polymers, a large pressure drop, called entrance loss, is encountered near an 
abrupt contraction, which can be used for indirect measurements of elongational viscosity. However, the flow near an abrupt contraction is not purely elongational in nature, but a mixture of shear and elongational flows. Therefore, an analysis based upon a purely elongational flow, such as the sink flow analysis of Metzner and Metzner [4], is expected to have a large error in the estimated entrance loss and elongational viscosity.

Cogswell [5] was the first one to perform a combined analysis of shear and elongational flows near an abrupt contraction. However, to simplify the analysis, Cogswell made a large number of simplifying assumptions. Binding [6] relaxed some of the assumptions of Cogswell and performed a more accurate analysis of entrance flow using the energy principle. The assumptions made by Cogswell and Binding were listed in Ref. 7. Cogswell and Binding's equations for axisymmetric and planar elongational viscosity, which were also given in Refs. 8 and 9, are summarized below.

Cogswell's equations:

$$
\begin{gathered}
\dot{\varepsilon}=\frac{k_{1} \tau_{\mathrm{w}} \dot{\gamma}_{\mathrm{a}}}{(n+1) P_{\mathrm{e}}} \\
\eta_{\mathrm{e}}=\frac{k_{2}(n+1) P_{\mathrm{e}}}{\dot{\varepsilon}}
\end{gathered}
$$

where $\dot{\varepsilon}$ is the elongation rate, $\eta_{\mathrm{e}}$ the elongational viscosity, $n$ the power law index for shear viscosity, $P_{\mathrm{e}}$ the entrance loss, $\tau_{\mathrm{w}}$ the shear stress at the wall in the downstream channel, and $\dot{\gamma}_{\mathrm{a}}$ is the apparent shear rate. Values of $\tau_{\mathrm{w}}, \dot{\gamma}_{\mathrm{a}}$, and the parameters $k_{1}$ and $k_{2}$ for axisymmetric and planar flows are given in Table 1 . The value of $k_{2}$ for planar flow in Table 1 is slightly different than the value given in Ref. 9. Beaupre [8] derived the equation for planar flow based upon Cogswell's analysis, and used the equation $\sigma_{\text {ave }}=\frac{4}{3} \eta_{\mathrm{e}} \dot{\varepsilon}$ for planar flows, which was given by Cogswell in Ref. 5. However, in this planar flow equation Cogswell still used axisymmetric elongational viscosity for $\eta_{\mathrm{e}}$, whereas it was interpreted as planar elongation viscosity by Beaupre [8], resulting in the erroneous values of parameter $k_{2}$ in Refs. 8 and 9.

TABLE 1. Cogswell analysis parameters for axisymmetric and planar flows.

\begin{tabular}{lcc}
\hline & Axisymmetric flow & Planar flow \\
\hline$k_{1}$ & $4 / 3$ & $2 / 3$ \\
$k_{2}$ & $3 / 8$ & $1 / 2$ \\
$\tau_{\mathrm{w}}$ & $\frac{\left(P_{\text {total }}-P_{\mathrm{e}}\right) r}{2 L}$ & $\frac{(\Delta P) H_{\mathrm{o}}}{L\left(1+\frac{H_{\mathrm{o}}}{W}\right)}$ \\
$\dot{\gamma}_{\mathrm{a}}$ & $\frac{3 Q}{\pi R_{\mathrm{o}}^{3}}$ & $\frac{Q}{W H_{\mathrm{o}}^{2}}$
\end{tabular}

TABLE 2. Binding's analysis parameters for axisymmetric and planar flows.

\begin{tabular}{lcc}
\hline & Axisymmetric & Planar \\
\hline$g_{1}$ & $2 / 3$ & $1 / 2$ \\
$g_{2}$ & $3 n+1$ & $2 n+1$ \\
$g_{3}$ & 3 & 2 \\
$g_{4}$ & 2 & 1 \\
$\alpha$ & $R_{\mathrm{o}} / R_{1}$ & $H_{\mathrm{o}} / H_{1}$ \\
$\dot{\gamma}_{\mathrm{w}}$ & $\frac{(3 n+1) Q}{\pi n R_{\mathrm{o}}^{3}}$ & $\frac{(2 n+1) Q}{2 W n H_{\mathrm{o}}^{2}}$ \\
\hline
\end{tabular}

Binding's equations:

For a power-law fluid with shear viscosity $\eta_{\mathrm{s}}=A(\dot{\gamma})^{n-1}$ and the elongational viscosity $\eta_{\mathrm{e}}=B(\dot{\varepsilon})^{m-1}$,

$$
P_{\mathrm{e}}=\frac{g_{1} A(1+m)^{2}}{m^{2}(1+n)^{2}}\left\{\frac{B m g_{2} n^{m} I_{n m}}{A}\right\}^{\frac{1}{1+m}} \dot{\gamma}_{\mathrm{w}}^{\frac{m(n+1)}{1+m}}\left\{1-\alpha^{\frac{g_{3} m(n+1)}{1+m}}\right\}
$$

where $P_{\mathrm{e}}$ is the entrance loss and $\dot{\gamma}_{\mathrm{w}}$ is the shear rate at the wall. Values of $\dot{\gamma}_{\mathrm{w}}$ and the parameters $g_{1}, g_{2}, g_{3}$, and $\alpha$, which are different for axisymmetric and planar flows, are given in Table 2 . In Table $2, R_{\mathrm{o}}\left(H_{\mathrm{o}}\right)$ and $R_{1}\left(H_{1}\right)$ are the radii (height) of the downstream and upstream channels, respectively, $Q$ is the flow rate, and $W$ is the width of the slit in the planar case. In $E q .3, I_{n m}$ is given by the following equation:

$$
I_{n m}=\int_{0}^{1}\left|g_{4}-\left(\frac{g_{2}}{n}\right) \phi^{1+\frac{1}{n}}\right|^{m+1} d \phi
$$

\section{PROCEDURE FOR ESTIMATION OF ELONGATIONAL VISCOSITY}

In the present work, PELDOM software [10] was used to estimate the elongational viscosity of four different polymers. The Carreau model was used for shear viscosity $\left(\eta_{\mathrm{s}}\right)[11]:$

$$
\eta_{\mathrm{s}}=\eta_{0}\left(1+\left(\lambda e_{i i}\right)^{2}\right)^{\frac{(n-1)}{2}}
$$

The model proposed by Sarkar and Gupta [12] was employed for elongational viscosity $\left(\eta_{\mathrm{e}}\right)$.

$$
\eta_{\mathrm{e}}=\eta_{0}\left[T_{\mathrm{r}}+\delta\left\{1-\frac{1}{\sqrt{1+\left(\lambda_{1} e_{i i}\right)^{2}}}\right\}\right]\left[1+\left(\lambda_{2} e_{i i}\right)^{2}\right]^{\frac{m-1}{2}}
$$

where $e_{\mathrm{ii}}=\sqrt{2 \tilde{e} \tilde{e}}$, the second invariant of the strain-rate tensor $(\tilde{e})$, is the same as shear rate, $\dot{\gamma}$, in the shear viscosity equation, whereas in the elongational viscosity equation $e_{i i}=\sqrt{3} \dot{\varepsilon}$ for axisymmetric flows and $e_{i i}=2 \dot{\varepsilon}$ for planar flows, with $\dot{\varepsilon}$ being the elongation rate. In Eq. $6, T_{\mathrm{r}}$, 
the Trouton ratio at low strain rates, is 3 for axisymmetric flows and 4 for planar flows, and $h_{\mathrm{o}}, \lambda, n, \delta, \lambda_{1}, \lambda_{2}$, and $m$ are the material parameters. It should be noted that the Sarkar-Gupta model (Eq. 6) can capture the elongationthickening followed by elongation-thinning behavior of elongational viscosity curve. Effect of the four elongational viscosity parameters $\left(\delta, \lambda_{1}, \lambda_{2}\right.$, and $\left.m\right)$ on the shape of the elongational viscosity curve was discussed in Ref. 7. In particular, parameter $\lambda_{1}$ specifies $1 / e_{i i}$ for transition between Newtonian and elongation-thickening portions of the viscosity strain-rate curve, whereas $\delta$ characterizes the total increase in viscosity in the elongation-thickening portion. Parameter $\lambda_{2}$ specifies $1 / e_{i i}$ for transition between the elongation-thickening and power-law region, and $m$ is the power-law index for elongational viscosity.

To capture the temperature dependency of shear and elongational viscosities, an Arrhenius type model was used for the zero-shear viscosity $\left(\eta_{\mathrm{o}}\right)$ in Eqs. 5 and 6.

$$
\eta_{0}=A \exp \left(\frac{T_{\mathrm{a}}}{T}\right)
$$

where, $T$ is the temperature of the polymer, and $A$ and $T_{\mathrm{a}}$ are material parameters. Besides the zero-shear viscosity, the parameters $\lambda, \lambda_{1}$, and $\lambda_{2}$ in Eqs. 5 and 6 also depend on temperature. Following the time-temperature superposition principle, values of these parameters at a specific temperature can be calculated using the equation given below.

$$
\lambda_{i}=\frac{\eta_{0}}{\eta_{0 \mathrm{a}}} \lambda_{\mathrm{ia}}
$$

where $\lambda_{i}$ is $\lambda, \lambda_{1}$, or $\lambda_{2}$, and $\eta_{\mathrm{o}}$ is the zero-shear viscosity at the same temperature at which $\lambda_{i}$ is to be calculated, and $\lambda_{i \mathrm{a}}$ and $\eta_{\mathrm{oa}}$ are the values of $\lambda_{i}$, and $\eta_{\mathrm{o}}$ at temperature $T_{\mathrm{a}}$.

PELDOM estimates the elongational viscosity of a polymer by optimizing the values of the four parameters, $\delta, \lambda_{1}, \lambda_{2}$, and $m$, in the elongational viscosity model $(E q$. $6)$, such that the difference between the entrance loss predicted by a finite element simulation and the corresponding experimental data for a range of flow rates in capillary or slit rheometer is minimized. The optimization scheme used in PELDOM for estimating the four elongation viscosity parameters was described in Ref. 7. PELDOM estimates the elongational viscosity for the temperature at which the capillary or slit rheometer experiments are conducted. In the present work, the shear and elongational viscosities were determined at two different temperatures for four polymers. The time-temperature superposition along with the Arrhenius model for the zero-shear viscosity ( $E q .7)$ was then used to determine the temperature dependence of viscosities. As expected, the values of power-law indices ( $n$ and $m$ ) and $\lambda_{i} / \eta_{\mathrm{o}}$ were slightly different for different temperatures. The values of these parameters for various polymers given later in this paper are the average values for each polymer.

\section{EQUIPMENT AND EXPERIMENTAL PROCEDURE}

A Goettfert Rheometer 3000 [13] was used at Datapoint Labs [14] to conduct the capillary and slit rheometer experiments. Capillary experiments were conducted with capillary diameter of $1 \mathrm{~mm}$ and capillary lengths of 5 and $20 \mathrm{~mm}$. For the planar flow, the height and width of the die opening in the slit die were 0.5 and $10 \mathrm{~mm}$, respectively. The slit die had a pressure transducer located in the reservoir, and three transducers in the slit. The transducers in the slit were located at a distance of 30,55 , and $80 \mathrm{~mm}$ from the slit entrance.

To determine the entrance loss for axisymmetric flow, Bagley plots [3] were prepared using the pressure data from the capillary experiments. The entrance loss was used to estimate the axisymmetric elongational viscosity as well as the Bagley correction for shear viscosity estimation. For the planar flow in the slit rheometer, the pressure readings from the three transducers in the slit were extrapolated to the slit entrance. The difference between the extrapolated pressure and the pressure reading from the transducer in the reservoir was used as the entrance loss for planar flows. For the capillary as well as slit rheometer data, Weissenberg-Rabinowitsch correction was applied to determine the true viscosity.

To measure the shear viscosity at low shear rates, an ARES parallel plate viscometer from TA Instruments [15] was used at The Dow Chemical Company. The dynamic viscosity experiments with the ARES viscometer were conducted using $25-\mathrm{mm}$ parallel plates at the same temperatures as those used for capillary and slit rheometer experiments. The combined data from the parallel plate viscometer and from capillary and slit rheometers was then used to fit the Carreau model parameters for shear viscosity.

The experiments were conducted for four different polymers. All four polymers used were from The Dow Chemical Company. Two of the four polymers were lowdensity polyethylenes with melt index of 8.0 (LDPE1) and 2.0 (LDPE2) g/10 $\mathrm{min}\left(190^{\circ} \mathrm{C}, 2.16 \mathrm{~kg}\right)$ and the other two polymers were polystyrenes with melt index of 5.0 (PS1) and 10.0 (PS2) $\mathrm{g} / 10 \mathrm{~min}\left(200^{\circ} \mathrm{C}, 5 \mathrm{~kg}\right)$. For the two LDPEs the experiments were performed at 200 and $215^{\circ} \mathrm{C}$, whereas 200 and $220^{\circ} \mathrm{C}$ temperatures were used for the two polystyrenes.

\section{RESULTS AND DISCUSSION}

For LDPE1, the entrance loss data from capillary and slit rheometers are shown in Figs. 1 and 2, respectively. On the log-log graphs, at higher flow rates the entrance loss increases almost linearly with flow rate. The deviation from this linear behavior at low flow rates in Figs. 1 and 2 may be due to larger error in the entrance loss data. The error in the entrance loss, which is determined as a small difference between two large numbers, generally increases at lower flow rates. 


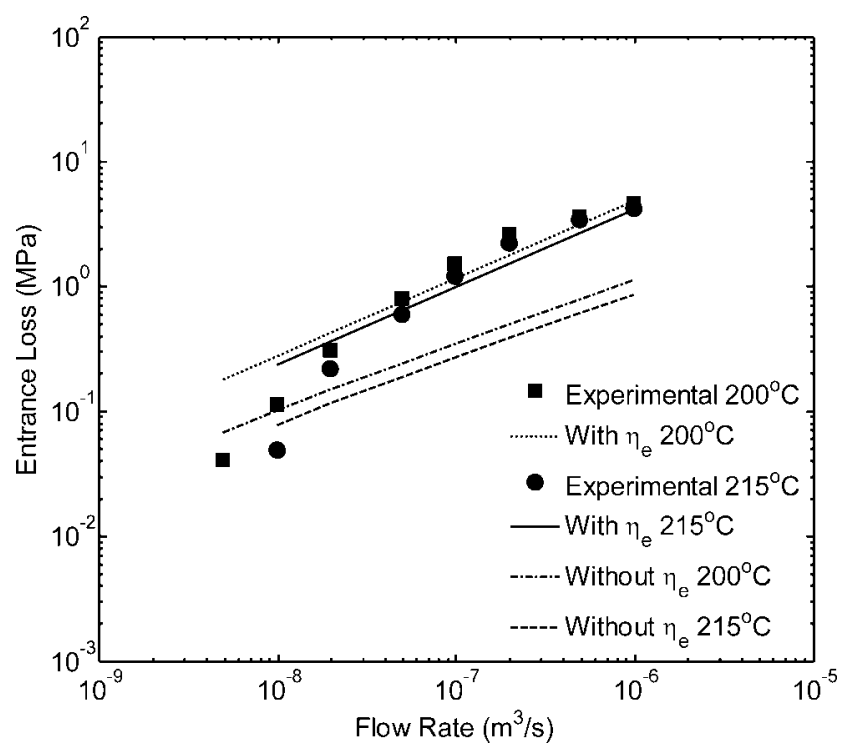

FIG. 1. Axisymmetric entrance loss for LDPE1.

The axisymmetric and planar elongational viscosities estimated using the entrance loss data in Figs. 1 and 2 and employing the elongation viscosity estimate procedure discussed earlier are shown in Figs. 3 and 4, respectively. Shear viscosity for LDPE1, which is also required for estimating the elongational viscosities, is also shown in Figs. 3 and 4. As mentioned earlier, shear viscosity at low shear rates was determined using a parallel plate viscometer, whereas at higher shear rates the viscosity was determined using capillary and slit rheometers. The data from parallel plate viscometer, which ranges for shear rates from $10^{-2}$ to $10^{2} \mathrm{~s}^{-1}$, is shown in Fig. 3 as well as in Fig. 4. The data from capillary rheometer $\left(\dot{\gamma}>1000 \mathrm{~s}^{-1}\right)$ is shown in Fig. 3 only, whereas the data from slit rheometer $\left(\dot{\gamma}>1000 \mathrm{~s}^{-1}\right)$ is shown in Fig. 4. The

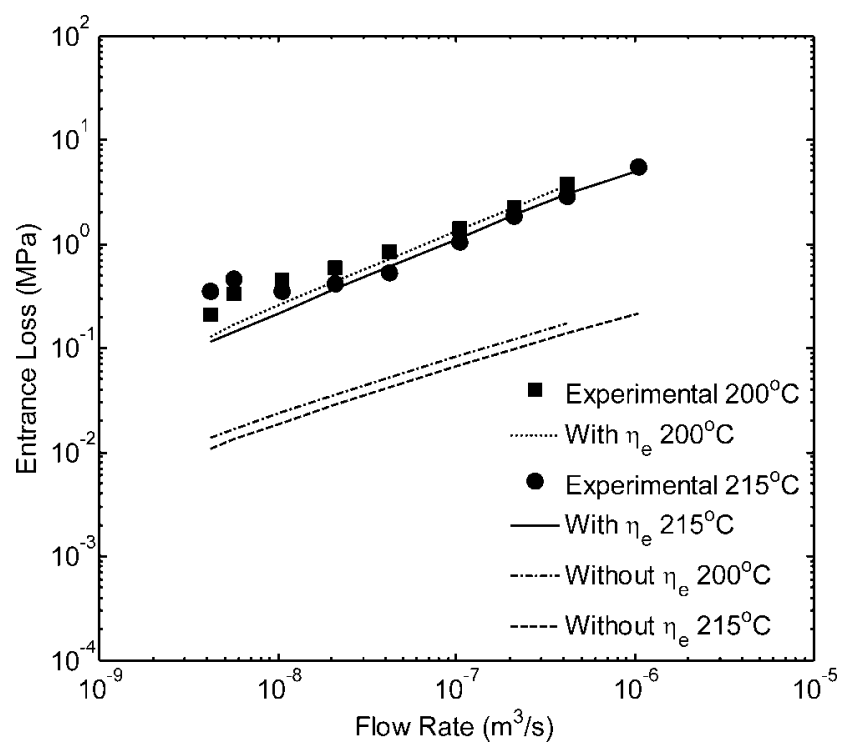

FIG. 2. Planar entrance loss for LDPE1.

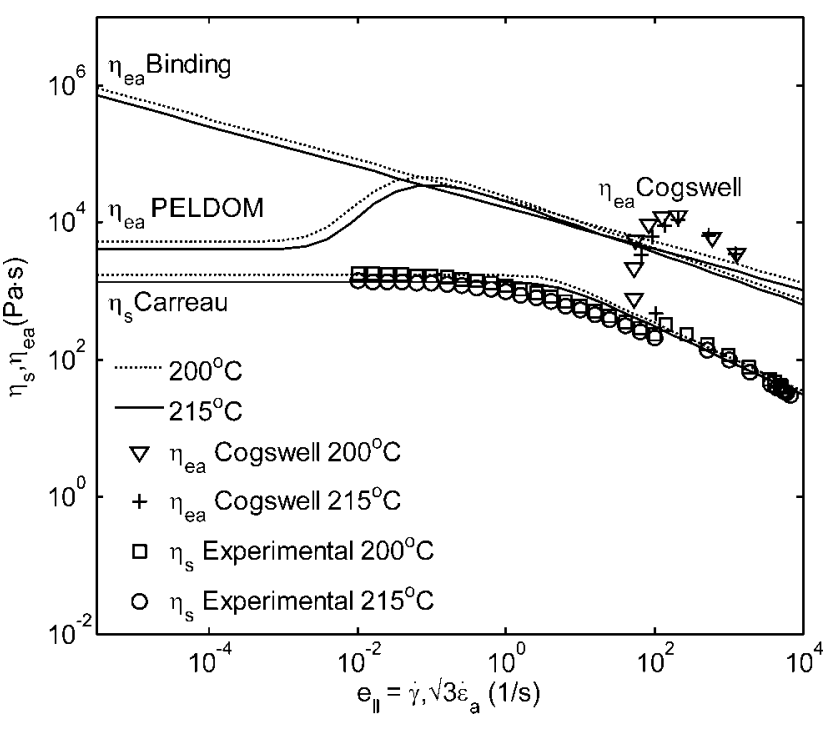

FIG. 3. Axisymmetric elongational viscosity of LDPE1.

combined shear viscosity data from all three sources was used to fit the Carreau model curves shown in Figs. 3 and 4. The Carreau model parameters for the shear viscosity curves in Figs. 3 and 4 are given in Table 3.

For LDPE2, The entrance loss and the shear and elongational viscosities are shown in Figs. 5-8. For the elongational viscosity curves in Figs. 3, 4, 7, and 8 the values of various parameters for the Sarkar-Gupta model for axisymmetric and planar elongational viscosities are given in Tables 4 and 5, respectively. At low strain rates, the axisymmetric and planar elongational viscosities are, respectively, 3 and 4 times the shear viscosity. At higher flow rates, even though they are different in magnitude, the predicted elongational viscosity curves for LDPE1 and LDPE2 in Figs. 3, 4, 7, and 8 have similar trends, and are also similar to those for LDPE $132 \mathrm{i}$ given in Ref. 9. In

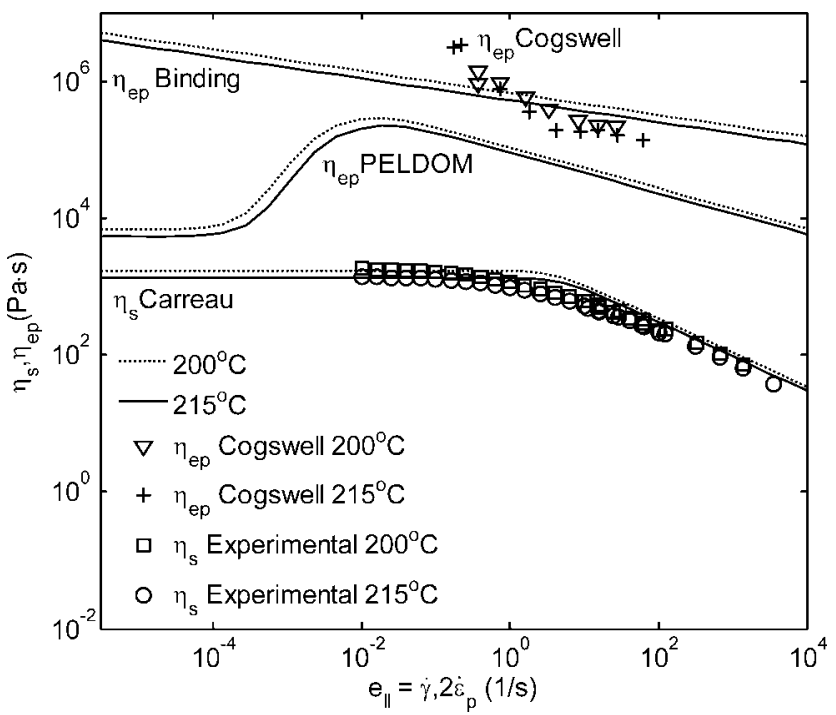

FIG. 4. Planar elongational viscosity of LDPE1. 
TABLE 3. Carreau model parameters for shear viscosity of the four polymers.

\begin{tabular}{lcccc}
\hline Material & $A(\mathrm{~Pa})$ & $T_{\mathrm{a}}(\mathrm{K})$ & $\lambda_{\mathrm{a}}(\mathrm{s})$ & $n$ \\
\hline LDPE1 & $5.39 \times 10^{-2}$ & $3.82 \times 10^{3}$ & $2.19 \times 10^{-4}$ & 0.50 \\
LDPE2 & $5.74 \times 10^{-5}$ & $8.71 \times 10^{3}$ & $1.55 \times 10^{-8}$ & 0.44 \\
PS1 & $1.59 \times 10^{-6}$ & $1.21 \times 10^{4}$ & $7.59 \times 10^{-10}$ & 0.31 \\
PS2 & $4.81 \times 10^{-6}$ & $1.09 \times 10^{4}$ & $2.77 \times 10^{-9}$ & 0.32 \\
\hline
\end{tabular}

particular, it is noted that for LDPE1, LDPE2, as well as LDPE 132i [9], the planar elongational viscosity is always higher than the axisymmetric elongational viscosity, which is larger than the shear viscosity. The axisymmetric as well as planar elongational viscosities of LDPE1 and LDPE2 exhibit an elongation-thickening region, which is followed by an elongation-thinning region at higher elongation rates. Similar behavior was observed for planar elongational viscosity of LDPE $132 \mathrm{i}$ in Ref. 9. However, the elongation-thickening region of axisymmetric elongational viscosity for LDPE $132 \mathrm{i}$ could not be captured in Ref. 9. For LDPE1 and LDPE2, the axisymmetric and planar elongational viscosities predicted by Cogswell's and Binding's analyses (Eqs. 1-4) are also shown in Figs. $3,4,7$, and 8. In general, for LDPE1 and LDPE2 axisymmetric and planar elongational viscosities predicted by the Cogswell's analysis are higher than the corresponding finite element based predictions from PELDOM. For the two LDPEs, predictions from Binding analysis for axisymmetric elongational viscosity are in good agreement with those from PELDOM, whereas for planar elongational viscosity the predictions are higher. Similar discrepancy between the predictions from Cogswell's and Binding's analyses and those from PELDOM was also observed for LDPE 132i in Ref. 9. For LDPE 132i in Ref. 9, the elongational viscosities predicted by Cogs-

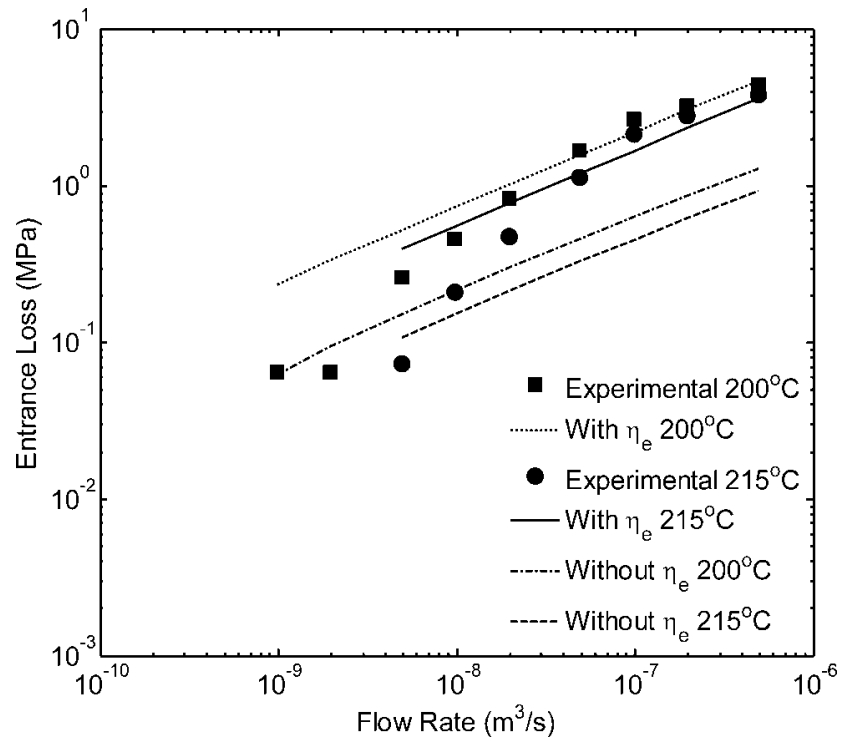

FIG. 5. Axisymmetric entrance loss for LDPE2.

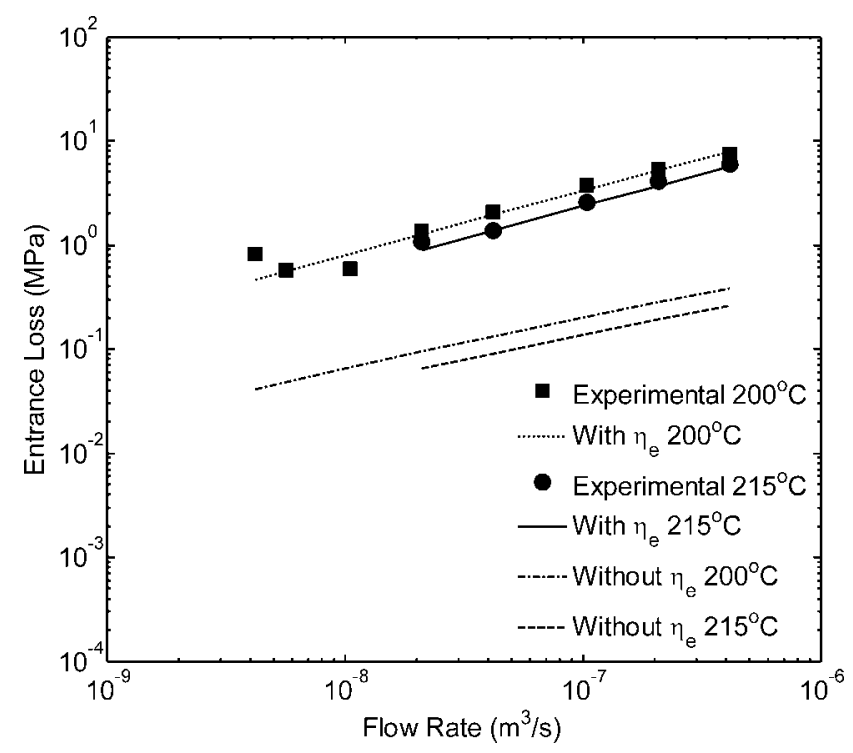

FIG. 6. Planar entrance loss for LDPE2.

well's analysis decreased almost linearly with elongation rate on a $\log -\log$ plot. However, in Figs. 3, 4, 7, and 8 the elongational viscosities for LDPE1 and LDPE2 predicted by Cogswell's analysis have some scatter. In particular, axisymmetric elongational viscosity predicted by Cogswell analysis first increases and then decreases as the elongation rate is increased. This unexpected behavior of elongational viscosity from Cogswell's analysis may be due to inaccuracy in the pressure measured at low flow rates. As discussed in Ref. 7, two entrance loss data points at higher flow rates are used in PELDOM to estimate the values of the two power-law parameters $\left(\lambda_{2}\right.$ and $m)$ in the Sarkar-Gupta model. The same two points were also used to estimate the elongational viscosity from the Binding's analysis. At the two different temperatures, the

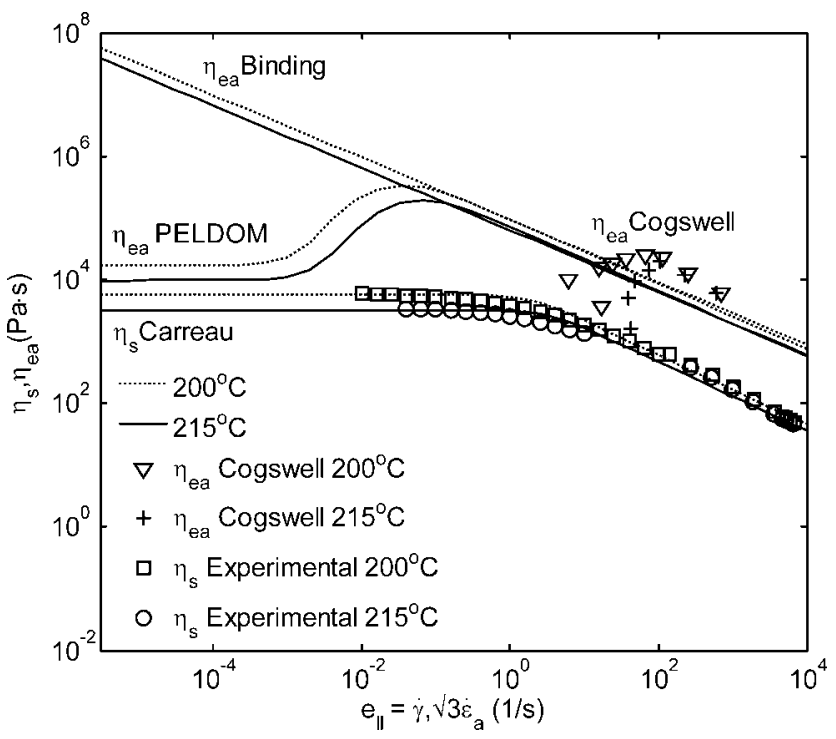

FIG. 7. Axisymmetric elongational viscosity of LDPE2. 


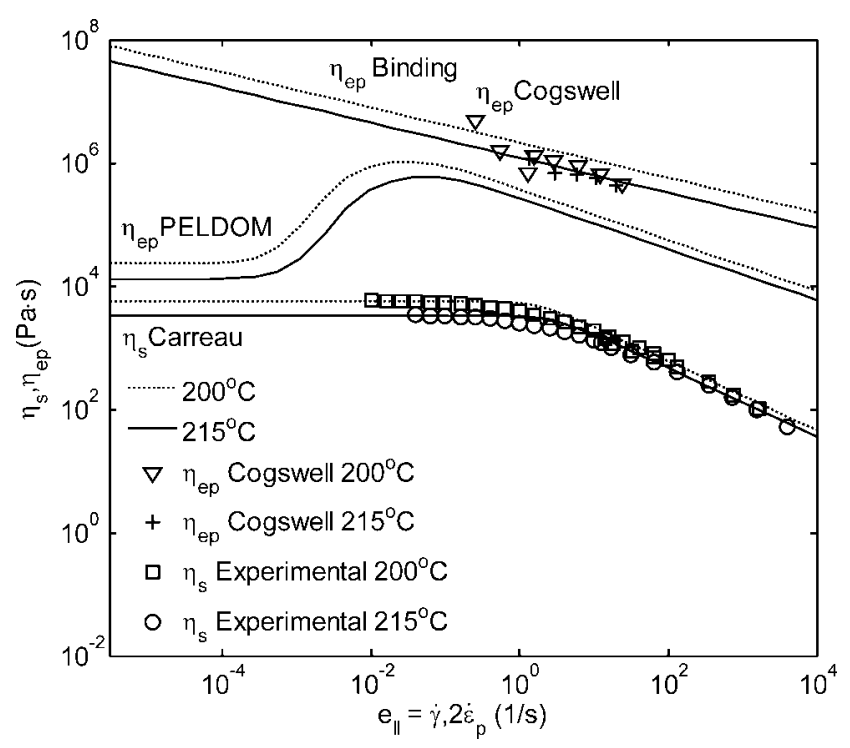

FIG. 8. Planar elongational viscosity of LDPE2.

values of $m$ predicted by Binding's analysis were slightly different. A similar difference was also found in the values of $m$ predicted by PELDOM. Therefore, an average value of $m$ for each resin at the two temperatures was used for the elongational viscosity given in this paper. Also, the values of $\lambda_{1}, \lambda_{2}$ in the Sarkar-Gupta model and that of $B$ in the power-law model for the elongational viscosity were averaged according to the time-temperature superposition.

At higher flow rates the experimental entrance loss data for LDPE1 and LDPE2 in Figs. 1, 2, 5, and 6 and the corresponding predictions by the PELDOM software including the elongational viscosity effects are in good agreement. The discrepancy between the entrance loss data at lower flow rates and the predictions from PELDOM again raises doubts about the accuracy of the experimental data at lower flow rates. Figures 1, 2, 5, and 6 also show the entrance loss data predicted by the Carreau model without including the effect of the high elongational viscosity of LDPEs. When the effect of the high elongational viscosity is ignored, for both LDPEs the predicted entrance loss is only about $20 \%$ of the experimental value for axisymmetric flow and about $10 \%$ of the experimental value for the planar flow. Similar errors were obtained for LDPE 132i also in Ref. 9 when the effect of high elongational viscosity was ignored in the simulation.

TABLE 4. Sarkar-Gupta model parameters for axisymmetric elongational viscosity of the four polymers.

\begin{tabular}{lcccc}
\hline Material & $\delta$ & $\lambda_{1 \mathrm{a}}(\mathrm{s})$ & $\lambda_{2 \mathrm{a}}(\mathrm{s})$ & $m$ \\
\hline LDPE1 & 29.0 & $8.22 \times 10^{-2}$ & $7.90 \times 10^{-3}$ & 0.62 \\
LDPE2 & 72.3 & $4.81 \times 10^{-6}$ & $4.89 \times 10^{-7}$ & 0.47 \\
PS1 & 0.00 & $1.44 \times 10^{14}$ & $3.48 \times 10^{-7}$ & 0.67 \\
PS2 & 0.00 & $3.77 \times 10^{14}$ & $1.48 \times 10^{-6}$ & 0.72 \\
\hline
\end{tabular}

TABLE 5. Sarka-Gupta model parameters for planar elongational viscosity of the four polymers.

\begin{tabular}{lcccc}
\hline Material & $\delta$ & $\lambda_{1 \mathrm{a}}(\mathrm{s})$ & $\lambda_{\text {2a }}(\mathrm{s})$ & $m$ \\
\hline LDPE1 & $1.87 \times 10^{-2}$ & $5.31 \times 10^{-1}$ & $3.60 \times 10^{-2}$ & 0.70 \\
LDPE2 & $2.10 \times 10^{-2}$ & $9.00 \times 10^{-6}$ & $4.96 \times 10^{-7}$ & 0.59 \\
PS1 & 65.95 & $1.24 \times 10^{-7}$ & $2.29 \times 10^{-8}$ & 0.57 \\
PS2 & 35.76 & $3.77 \times 10^{-8}$ & $3.53 \times 10^{-9}$ & 0.47 \\
\hline
\end{tabular}

For estimation of axisymmetric elongational viscosity, dies with different capillary lengths were used to estimate the entrance loss with the only pressure transducer being located in the reservoir. Therefore, it is difficult to compare the predicted pressure distribution in the axisymmetric entrance flow with the experimental data. However, for the planar flow, three pressure transducers were located in the slit die and one transducer was located in the reservoir. For the two LDPEs the pressure recorded from these four transducers and the corresponding predictions in the planar entrance flow are shown in Fig. 9. For both LDPEs, the pressure distributions in Fig. 9 are for the flow rate of $2.88 \times 10^{-7} \mathrm{~m}^{3} / \mathrm{s}$. To estimate the entrance loss, as shown in Fig. 9, the pressure drop in the reservoir is neglected and a linear fit to the pressure data in the slit is extrapolated until the slit entrance. For LDPE1 as well as LDPE2 the predicted entrance loss $\left(\Delta P_{\mathrm{e}}\right)$ including elongational viscosity effects is in good agreement with the experimental data. However, for LDPE1 the predicted pressure is significantly higher than the experimental data. The reason for the difference between the prediction and the experimental data for LDPE1 is evident from the viscosity plots in Figs. 3 and 4. As mentioned earlier, the combined shear viscosity data

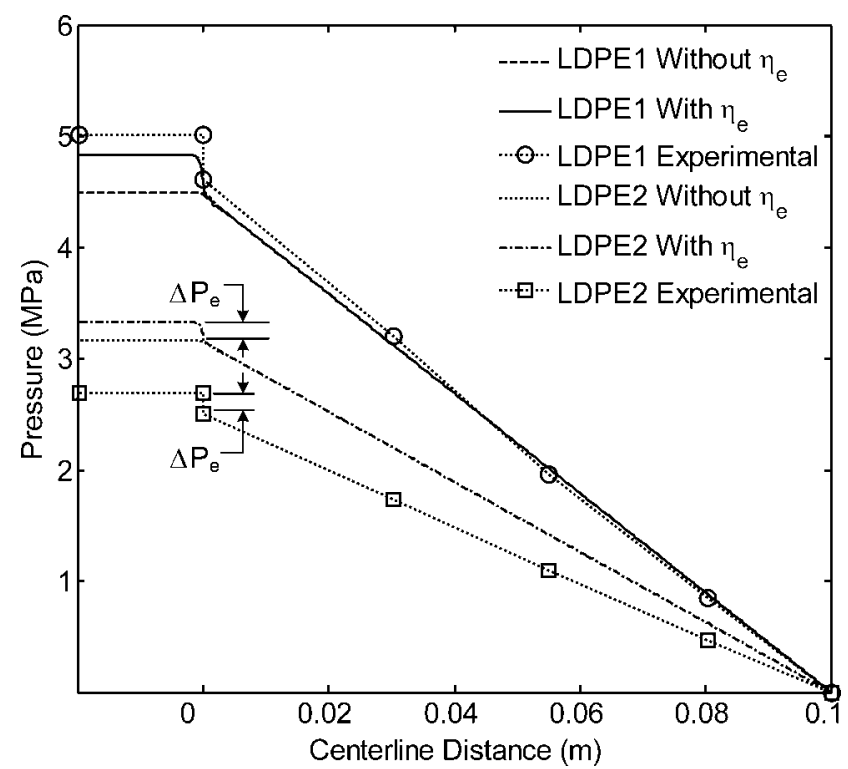

FIG. 9. Pressure distributions along the slit die for LDPE1 and LDPE2. The slit entrance is located at centerline distance of zero, and $\Delta P_{\mathrm{e}}$ is the entrance loss. 


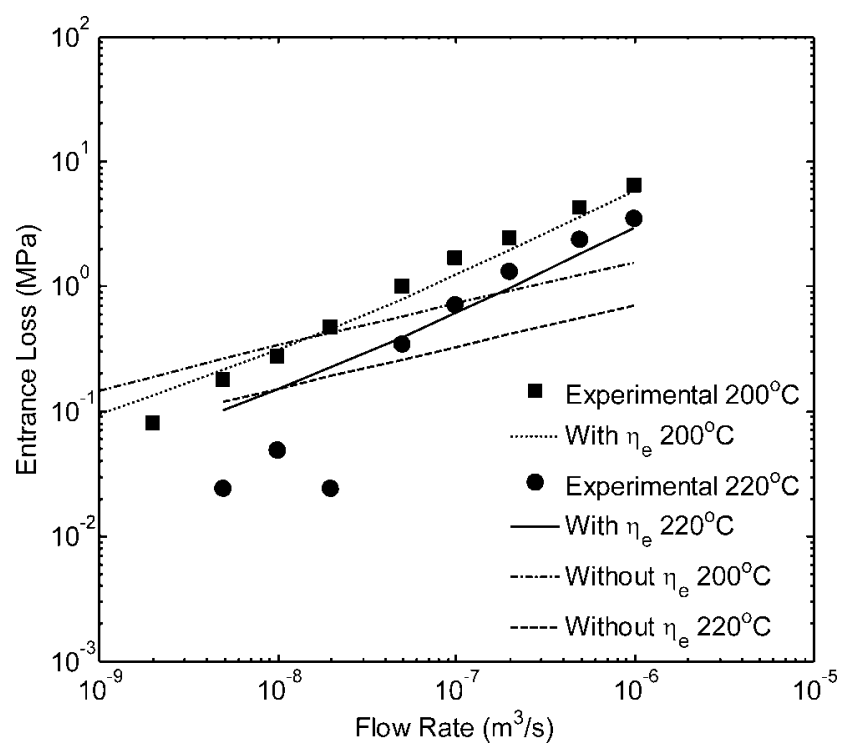

FIG. 10. Axisymmetric entrance loss for PS1.

from capillary and slit rheometers was used to fit the Carreau model parameters. In Fig. 4, the viscosity from the Carreau model is slightly higher than the viscosity data from the slit rheometer. Since the shear viscosity from the Carreau model is higher than the viscosity data from the slit rheometer, as expected, the pressure in the slit die predicted by the finite element simulation of the entrance flow for LDPE1 is higher than the corresponding experimental data. Since the shear viscosity for LDPE2 from the capillary and slit rheometer in Figs. 7 and 8, respectively, are in good agreement and match accurately with Carreau model, the predicted pressure variation for LDPE2 in Fig. 9 is in good agreement with the experimental data. It should also be noted that in Fig. 9, for LDPE1, as well as LDPE2, entrance loss predicted with-

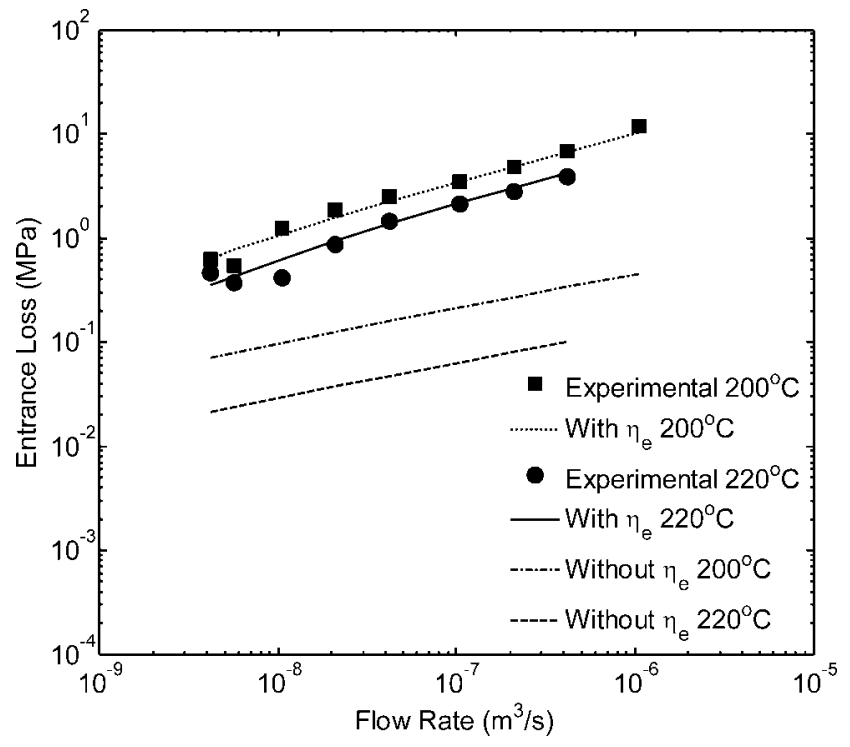

FIG. 11. Planar entrance loss for PS1.

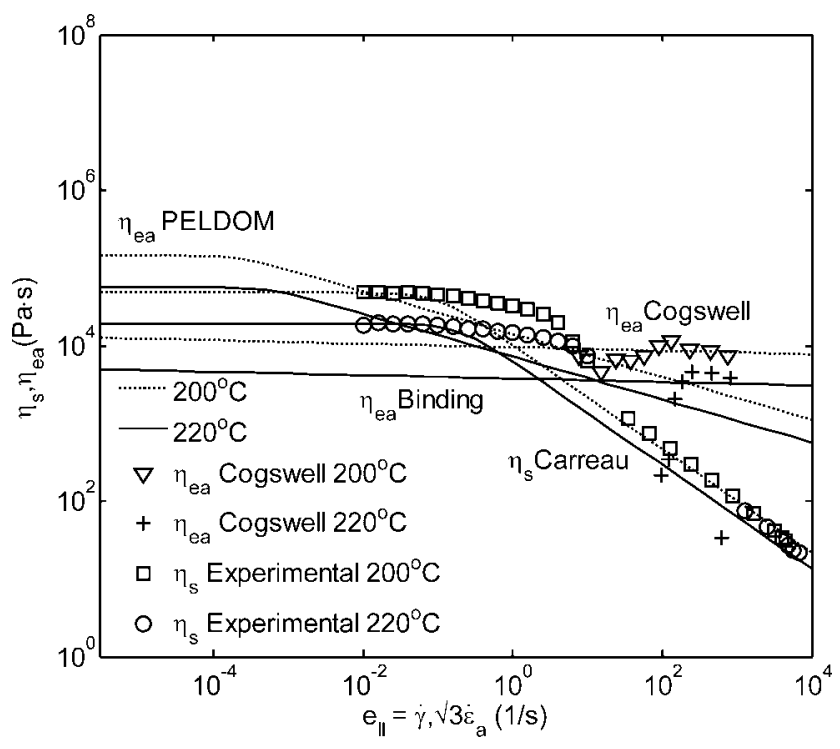

FIG. 12. Axisymmetric elongational viscosity of PS1.

out elongational viscosity effects is much smaller than the entrance loss obtained in experimental data. Also, since the flow in the slit die is purely shearing in nature, in Fig. 9 , elongational viscosity has no effect on the predicted pressure down stream of the abrupt contraction, that is, in the slit die.

The entrance loss data and the estimated elongational viscosity for PS1 and PS2 are shown in Figs. 10-17. Again, the shear viscosity data at low shear rate is the dynamic viscosity data from a parallel plate viscometer, whereas the higher shear rate data in Figs. 12 and 13 is from capillary and slit rheometers, respectively.

The planar elongational viscosities of PS1 and PS2 in Figs. 13 and 17 show variation similar to that of the planar elongational viscosities of LDPEs in Figs. 4 and 8.

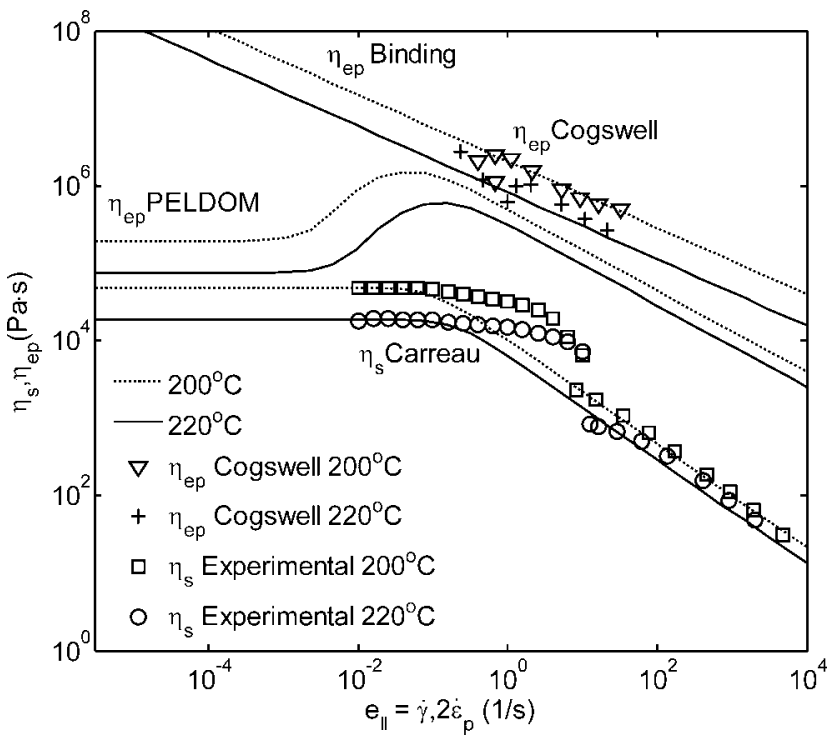

FIG. 13. Planar elongational viscosity of PS1. 


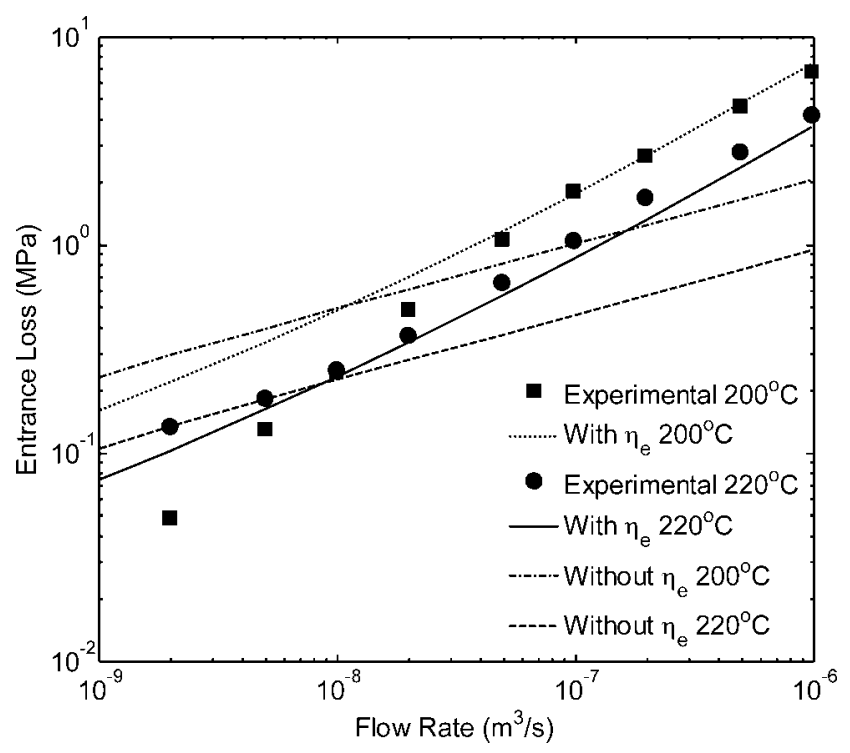

FIG. 14. Axisymmetric entrance loss for PS2.

For the two polystyrenes, the values of various parameters for the Sarkar-Gupta elongational viscosity model are also given in Tables 4 and 5. The planar elongational viscosities for the two polystyrenes also go through an elongation-thickening region, which is followed by a decrease in the elongational viscosity. Also, the planar elongational viscosity of the two polystyrenes is higher than the axisymmetric elongational viscosity and the shear viscosity. In contrast, the estimated axisymmetric elongational viscosity of polystyrenes in Figs. 12 and 16 does not have any elongational-thickening region. Unexpectedly, the axisymmetric elongational viscosities of polystyrenes at intermediate elongation rates $\left(0.01-1 \mathrm{~s}^{-1}\right)$ is below their shear viscosities. The elongational viscosity of polystyrene predicted by the Cogswell's analysis has similar

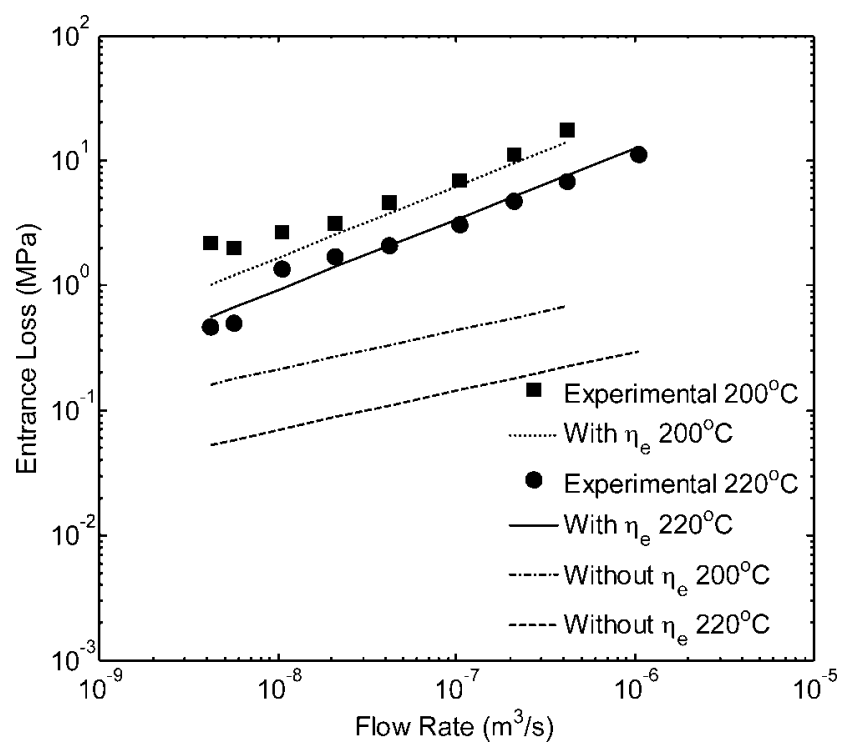

FIG. 15. Planar entrance loss of PS2.

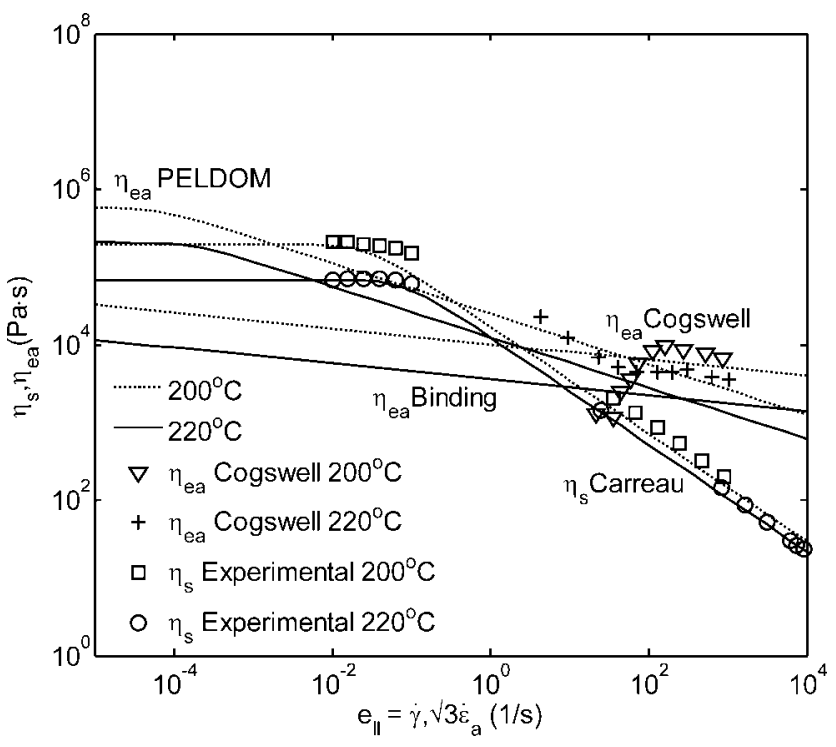

FIG. 16. Axisymmetric elongational viscosity of PS2.

behavior as that of the LDPEs. The predictions from Cogswell's analysis are higher than the corresponding predictions from the PELDOM software. Also, at lower elongation rates, the axisymmetric elongational viscosity predicted by Cogswell's analysis is unexpectedly lower than the linear behavior at higher elongation rates. In Figs. 13 and 17, planar elongational viscosity predicted by Binding analysis is also higher than the corresponding predictions from PELDOM. However, the planar elongational viscosity curves from the two analyses have similar slopes in the power-law region. In contrast, for the axisymmetric flow in Figs. 12 and 16, the slopes of the elongational viscosity curves from the two analyses are quite different.

In Figs. 10, 11, 14, and 15, the entrance loss predicted by the PELDOM software including the elongational vis-

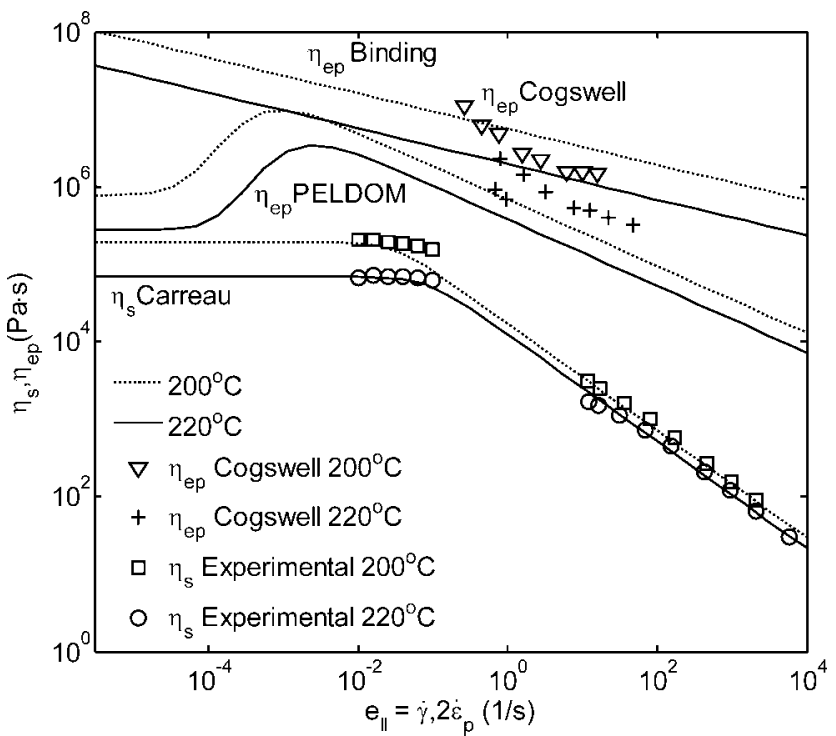

FIG. 17. Planar elongational viscosity of PS2. 


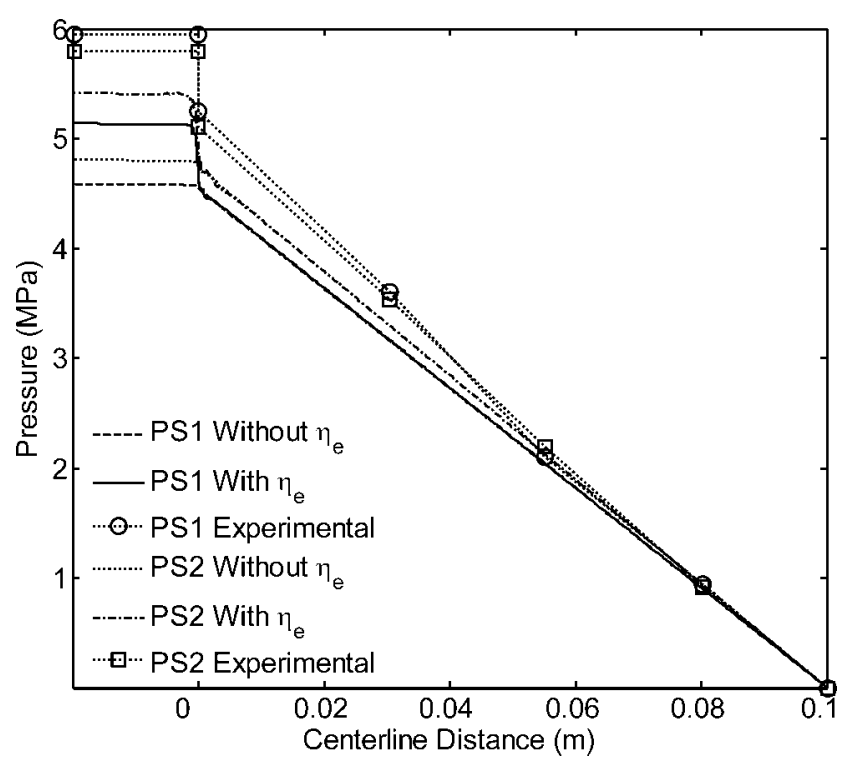

FIG. 18. Pressure distributions along the slit die for PS1 and PS2.

cosity effects is in good agreement with the experimental data at higher flow rates. However, the agreement is not as good at lower flow rates, particularly for the axisymmetric flow data from the capillary rheometer. Since the estimated planar elongational viscosity of the two polystyrenes at all elongational rates was higher than that for the shear thinning fluids using the Carreau model (which is four times the shear viscosity at all elongation rates), the entrance loss predicted by PELDOM in Figs. 11 and 15 is higher than the corresponding predictions using Carreau model without including the effect of higher elongational viscosity. At intermediate elongation rates, the axisymmetric elongational viscosity of the two polystyrenes predicted by PELDOM software was lower than that for shear-thinning fluids using the Carreau model (which is three times the shear viscosity at all elongation rates). Therefore, at lower flow rates in Figs. 10 and 14, the entrance loss predicted by PELDOM software is lower than that predicted by the Carreau model without elongational viscosity effects. The entrance loss predicted by PELDOM software in Figs. 10 and 14 is larger at higher flow rates, because at higher elongational rates the axisymmetric elongational viscosity predicted by PELDOM in Figs. 12 and 16 is larger than the elongational viscosity for shear-thinning fluids using Carreau model.

For the two polystyrenes, Fig. 18 compares the predicted pressure distribution in the slit rheometer with the corresponding experimental data. The pressure distribution for PS1 and PS2 are for flow rates of $1.04 \times 10^{-7}$ and $4.17 \times 10^{-7} \mathrm{~m}^{3} / \mathrm{s}$, respectively. As was observed for LDPEs in Fig. 9, for the two polystyrenes in Fig. 18, downstream of the abrupt contraction, the predicted pressure in the slit die with elongational effects included in the simulation is the same as the pressure without the elongation viscosity effects. But, the entrance loss pre- dicted with the elongational viscosity effect included in the simulation is in good agreement with the experimental data, whereas without elongational viscosity effects, the predicted entrance loss is much smaller than the experimental value. Even though the predicted entrance loss with elongational viscosity effects included in the simulation matches well with the experimental data, the pressure predicted is lower than the experimental value for both polystyrenes. Again, the reason for this disagreement is the difference in the shear viscosity from the Carreau model and the viscosity measured from the slit die experiments. In Figs. 13 and 17 for both polystyrenes, the viscosity from the Carreau model is slightly lower than the viscosity data from the slit rheometer, resulting in the lower pressure predictions in Fig. 18.

As discussed in Ref. 7, the elongational viscosities reported here should be treated as estimates of resistance to elongational deformation. Because of simplifications involved in the flow simulation, for instance, no account of the first and second normal stress differences, the estimated elongational viscosity may be some what different from the intrinsic elongational viscosity of the polymer. However, because of various difficulties involved in direct measurements of elongational viscosity at higher elongational rates, the current method is an excellent alternative for elongational viscosity estimation. The elongational viscosity estimations obtained here, even though approximate, are useful because they can be used to improve the predications from simulations of more complex threedimensional flows. Such an example for the use of estimated elongational viscosity to simulate a three-dimensional flow was presented in Ref. 16, where the flow of LDPE $132 \mathrm{i}$ in an extrusion die was simulated.

\section{CONCLUSIONS}

The planar and axisymmetric elongational viscosities of two LDPEs and polystyrenes were estimated by minimizing the difference between the entrance loss predicted by a finite element simulation and the corresponding data from slit and capillary rheometers, respectively. For each of the four polymers, elongational viscosities were estimated at two different temperatures. Planar elongational viscosity of all four polymers exhibited an elongationthickening region, which was followed by an elongationthinning region. Similar variation was obtained in the axisymmetric elongational viscosity of the two LDPEs. However, the elongation-thickening behavior could not be captured for the axisymmetric elongational viscosity of the two polystyrenes. Unexpectedly, at intermediate elongation rates, axisymmetric elongational viscosities of the two polystyrenes were below their shear viscosity.

\section{REFERENCES}

1. C.W. Macosko, Rheology Principles, Measurements, and Applications, VCH, New York, 240 (1994). 
2. F.T. Trouton, Proc. R. Soc. Lond. Ser. A, 77, 426 (1906).

3. F.A. Morrison, Understanding Rheology, Oxford University Press, New York, 382 (2001).

4. A.B. Metzner and A.P. Metzner, Rheol. Acta, 9, 174 (1970).

5. F.N. Cogswell, Polym. Eng. Sci., 12, 64 (1972).

6. D.M. Binding, J. Non-Newtonian Fluid Mech., 27, 193 (1988).

7. M. Gupta, Adv. Polym. Technol., 21, 98 (2002).

8. P. Beaupre, M.S. Thesis, A comparison of the axisymmetric \& planar elongational viscosity of a polymer. Michigan Technological University, Houghton (2002).

9. P. Beaupre and M. Gupta, Int. Polym. Proc., 17, 370 (2002).
10. PELDOM Software, Plastic Flow, LLC, 1206 Birch Street, MI 49931, USA.

11. P.J. Carreau, Ph.D. Thesis, Rheological equations from molecular network theories. University of Wisconsin, Madison (1968).

12. D. Sarkar and M. Gupta, J. Reinforced Plast. Compos., 20, 1473 I(2001).

13. Goettfert Inc., 488 Lakeshore Parkway, Rock Hill, SC 29730, USA.

14. Datapoint Labs, 95 Brown Road, Ithaca, NY 14850, USA.

15. TA Instruments, 109 Lukens Drive, New Castle, DE 19720.

16. Y. Sun, M. Gupta, J. Dooley, K.A. Koppi, and M.A. Spalding, SPE ANTEC Tech. Pap., 51, 81 (2005). 